\title{
Spin Transverse Force on Spin Current in an Electric Field
}

\author{
Shun-Qing Shen \\ Department of Physics, and Center for Theoretical and Computational Physics, The University of Hong Kong, \\ Pokfulam Road, Hong Kong, China \\ (Received 27 June 2005; published 25 October 2005)
}

\begin{abstract}
As a relativistic quantum mechanical effect, it is shown that the electron field exerts a transverse force on an electron spin $1 / 2$ only if the electron is moving. The spin force, analogue to the Lorentz for an electron charge in a magnetic field, is perpendicular to the electric field and the spin current whose spin polarization is projected along the electric field. This spin-dependent force can be used to understand the Zitterbewegung of the electron wave packet with spin-orbit coupling and is relevant to the generation of the charge Hall effect driven by the spin current in semiconductors.
\end{abstract}

In recent years, spintronics has become an emerging field because of its potential application to the semiconductor industry, and more and more attention is focused on the effect of spin-orbit coupling in metals and semiconductors [1]. The spin-orbit coupling is a relativistic effect describing the interaction of the electron spin, momentum, and electric field, and provides a route to manipulate and to control the quantum spin states via external fields $[2,3]$. It is desirable to understand the motion of electron spin with spin-orbit coupling in an electromagnetic field. In electrodynamics it is known that a magnetic field would exert a Lorentz force on an electric charge if it were moving. This Lorentz force can generate a lot of fundamental phenomena such as the Hall effect in solids [4]. The interaction of the spin in the electromagnetic field behaves as if the spin is a gauge charge and the interaction is due to the $\mathrm{SU}(2)$ gauge field [5]. It is essential that the electron spin is an intrinsic quantum variable, not a just a classical tiny magnetic moment. The physical meaning of the interaction is closely associated to the Aharonov-Casher effect and the Berry phase.

In this Letter it is found that an electric field exerts a transverse force on an electron spin if it is moving and the spin is projected along the electric field. The force is proportional to the square of electric field and the spin current projected along the field, and its direction is always perpendicular to the electric field and the speed of the spin. The force stems from the spin-orbital coupling which can be derived from the Dirac equation for an electron in a potential in the nonrelativistic limit or the Kane model with the $\mathbf{k} \cdot \mathbf{p}$ coupling between the conduction band and valence band. From an exact solution of a single electron with the spin-orbit coupling it is heuristic to understand that the Zitterbewegung of electronic wave packet is driven by the spin transverse force on a moving spin. The role of spin transverse force is also discussed in the spin Hall effect and its reciprocal effect driven by the pure spin current in semiconductors.

We start with the Dirac equation of an electron in a confining potential $V$ and a vector potential $\mathbf{A}$ for a mag- netic field, $\mathbf{B}=\nabla \times \mathbf{A}$,

$$
i \hbar \frac{\partial}{\partial t} \Psi=\left[c \alpha \cdot\left(\mathbf{p}+\frac{e}{c} \mathbf{A}\right)+m c^{2} \gamma+V\right] \Psi,
$$

where $\alpha$ and $\gamma$ are the $4 \times 4$ Dirac matrices. $m$ and $e$ are the electron mass and charge, respectively, and $c$ is the speed of light. We let $\Psi=\left(\begin{array}{l}\varphi \\ \chi\end{array}\right) e^{-i m c^{2} t / \hbar}$ such that the rest mass energy of electron is removed from the energy eigenvalue of the electron. In the nonrelativistic limit, $\chi$ is a very small component, $\chi \approx \frac{1}{m c^{2}-V} c \sigma \cdot\left(\mathbf{p}+\frac{e}{c} \mathbf{A}\right) \varphi$ where $\sigma$ are the Pauli matrices. Thus the component $\varphi$ of the wave function satisfies the following equation, $i \hbar \frac{\partial}{\partial t} \varphi \approx$ $H \varphi$, where

$$
H \approx \frac{\left(\mathbf{p}+\frac{e}{c} \mathbf{A}\right)^{2}}{2 m}+V_{\mathrm{eff}}+\mu_{B} \sigma \cdot \mathbf{B}+\frac{\hbar\left(\mathbf{p}+\frac{e}{c} \mathbf{A}\right)}{4 m^{2} c^{2}} \cdot(\sigma \times \nabla V),
$$

where $\mu_{B}=e \hbar / 2 m c$ and $V_{\text {eff }}=V+\frac{\hbar^{2}}{8 m^{2} c^{2}} \nabla^{2} V$. In the last step we neglect the higher order terms of expansion. Thus the Dirac equation is reduced to the Schrödinger equation with the spin-orbit coupling. The same form of effective spin-orbit coupling and Zeeman splitting can be also derived from the $8 \times 8$ Kane model that takes into account only the $\mathbf{k} \cdot \mathbf{p}$ coupling between the $\Gamma_{6}^{c}$ conduction band and the $\Gamma_{8}^{v}$ and $\Gamma_{7}^{v}$ valence bands, although the effective mass, the effective Lande $g$ factor, and the effective coupling coefficients have to be introduced as materialspecific parameters such as $m \rightarrow m^{*}, \mu_{B} \rightarrow g \mu_{B} / 2$, and $\hbar^{2} /\left(4 m^{2} c^{2}\right) \rightarrow r_{41}^{6 c 6 c}[6]$.

In the Heisenberg picture the kinetic velocity is

$$
\mathbf{v}=\frac{1}{i \hbar}[\mathbf{r}, H]=\frac{1}{m}\left[\mathbf{p}+\frac{e}{c}(\mathbf{A}+\mathcal{A})\right]
$$

where $\mathcal{A}=\frac{\hbar}{4 m c e} \sigma \times \nabla V$ and comes from the spin-orbit coupling. It indicates clearly that $\mathcal{A}$ plays a role of a $\mathrm{SU}(2)$ gauge vector potential. The spin dependence of the gauge field can separate the charged carriers with different spins in cyclotron motion experimentally [7]. Even though we have $\left[p_{\alpha}, p_{\beta}\right]=0$ for canonical momentum, 
the analogous commutators do not vanish for the kinetic velocity

$$
\left[v_{\alpha}, v_{\beta}\right]=-i \frac{\hbar e}{m^{2} c} \epsilon_{\alpha \beta \gamma} \mathcal{B}_{\gamma}+\frac{e^{2}}{m^{2} c^{2}}\left[\mathcal{A}_{\alpha}, \mathcal{A}_{\beta}\right]
$$

where the total magnetic field $\mathcal{B}=\mathbf{B}+\nabla \times \mathcal{A}$, and $\nabla \times$ $\mathcal{A}=\hbar\left[\sigma \cdot\left(\nabla^{2} V\right)-(\sigma \cdot \nabla) \nabla V\right] /(4 m c e)$. Notice that $\left[\mathcal{A}_{\alpha}, \mathcal{A}_{\beta}\right]=2 \hbar^{2} i(\sigma \cdot \nabla V) \epsilon_{\alpha \beta \gamma} \partial_{\gamma} V /(4 m c e)^{2}$. We can derive the quantum mechanical version of the force,

$$
m \frac{d \mathbf{v}}{d t}=F_{h}+F_{g}+F_{f}
$$

with

$$
\begin{aligned}
& F_{h}=-\frac{e}{c}(\mathbf{v} \times \mathcal{B}-\mathcal{B} \times \mathbf{v}) / 2-\nabla\left(V_{\mathrm{eff}}+\mu_{B} \sigma \cdot \mathbf{B}\right), \\
& F_{g}=\frac{\mu_{B}}{2 m c^{2}}[\sigma(\mathbf{B} \cdot \nabla V)-\mathbf{B}(\sigma \cdot \nabla V)], \\
& F_{f}=\frac{\hbar}{8 m^{2} c^{4}}(\sigma \cdot \nabla V)(\mathbf{v} \times \nabla V) .
\end{aligned}
$$

This is the quantum mechanical analogue of Newton's second law. Of course we should notice that this is just an operator equation. The uncertainty relationship tells us that the position and momentum cannot be measured simultaneously, and there is no concept of force in quantum mechanics. To see the physical meaning of the equation, we take the expectation values of both sides with respect to a Heisenberg state $|\Phi\rangle$ which does not vary with time. The expectation values of the observable describe the motion of the center of the wave package of electrons. In this sense we have an equation of the force experienced by the moving electron. Actually the first term $F_{h}$ in Eq. (5) is the Lorentz force for a charged particle in a magnetic field $\langle\mathcal{B}\rangle$ which contains the contribution from the $\mathrm{SU}(2)$ gauge field $\mathcal{A}$ as well as the conventional electromagnetic field. We have recovered the Ehrenfest theorem as one of the examples of the corresponding principle in quantum mechanics. The term, $\left\langle\nabla\left(\mu_{B} \sigma \cdot B\right)\right\rangle$, results from the nonuniform magnetic field. Its role was first realized in the SternGerlach experiment, where a shaped magnet is used to generate a nonuniform magnetic field to split the beam of silver atoms. In the classical limit it is written as the interaction between the magnetic momentum $\mu=$ $-\left\langle\mu_{B} \sigma\right\rangle$ and magnetic field. This spin force depends on the spin. Recently it is proposed that the force can generate a pure spin current if we assume $\nabla B_{z}$ is a constant [8]. In the term $F_{g}$ we can also use $\mu$ to replace the spin. It is nonzero only when the electric and magnetic fields coexist, as suggested by Anandan and others [5]. This term will play an essential role in generating spin Hall current in two-dimensional Rashba systems, which we will discuss it later. The last term, $F_{f}$, comes from the $\mathrm{SU}(2)$ gauge potential or spin-orbital coupling. As the force is related to the Planck constant it has no counterpart in classical mechanics and is purely quantum mechanic effect. The force is irrelevant of the magnetic field. In the classical limit we cannot simply use the magnetic momentum $\mu$ to replace the spin $\sigma$ in the potential $\mathcal{A}$. Otherwise the force vanishes. To see the physical meaning of the force, we write the spin force for a single electron on a quantum state in a compact form,

$$
\left\langle F_{f}\right\rangle=\frac{e^{2}|\mathcal{E}|}{4 m^{2} c^{4}} \mathbf{j}_{s}^{\mathcal{E}} \times \mathcal{E},
$$

where the spin current is defined conventionally, $\mathbf{j}_{s}^{\mathcal{E}}=\frac{\hbar}{4}\langle\{\mathbf{v}, \sigma \cdot \mathcal{E} /|\mathcal{E}|\}\rangle$, and in the last step the relation $\{\mathcal{A}, \sigma \cdot \mathcal{E}\}=0$ has been used. This is the main result in this Letter. The force is proportional to the square of electric field $\mathcal{E}$ and the spin current whose polarization is projected along the field. It is important to note that an electron in a spin state perpendicular to the electric field will not experience any force. Comparing with a charged particle in a magnetic field, $\mathbf{j}_{c} \times \mathbf{B}$, where $\mathbf{j}_{c}$ is a charge current density, the spin force is nonlinear to the electric field and depends on the spin state of electron.

We discuss several examples relevant to the spin force. Though the gauge field $\mathcal{A}$ provides a spin-dependent magnetic field $\nabla \times \mathcal{A}$, the Lorentz force caused by the field on the charge will vanish in a uniform electric field $\nabla V=e \mathcal{E}$. The spin dependent force $F_{g}$ also vanishes in the absence of magnetic field. Here we consider the motion of an electron confined in a two-dimensional plane subjected to a perpendicular electric field,

$$
H=\frac{\mathbf{p}^{2}}{2 m}+\lambda\left(\mathbf{p}_{x} \sigma_{y}-\mathbf{p}_{y} \sigma_{x}\right),
$$

where $\lambda=\hbar e \mathcal{E} /\left(4 m^{2} c^{2}\right)$ from Eq. (2). This can be regarded as counterpart of a charged particle in a magnetic field. On the other hand it has the same form of the Rashba coupling in a semiconductor heterojuction with the structural inversion asymmetry [9], where the spin-orbit coupling is induced by the offsets of valence bands at the interfaces and the structure inversion asymmetry [6]. A typical value of this coefficient $\lambda$ is of order $10^{-4} c$ ( $c$ the speed of light), and can be adjusted by an external field [10]. Because of the spin-orbit coupling the electron spin will precess with time,

$$
\frac{d \sigma(t)}{d t}=\frac{2 \lambda}{\hbar} \sigma(t) \times(\mathbf{p} \times \hat{z}) .
$$

Since the momentum $\mathbf{p}$ is a good quantum number, without loss of generality, we take $p=p_{x}$, just along the $x$ direction. Correspondingly the wave function in the position space $\langle r \mid \Phi\rangle=\exp \left(i p_{x} x\right) \chi_{s} / \sqrt{L}$ where $\chi_{s}$ is the initial spin state. Equivalently the spin-orbit coupling provides an effective magnetic field along the $y$ direction, $\mathbf{B}_{\text {eff }}=\lambda \mathbf{p}_{x} \hat{y}$. This problem can be solved analytically, and the electron spin precesses in the spin $x-z$ plane [11], $\sigma_{x}(t)=\sigma_{x} \cos \omega_{c} t-\sigma_{z} \sin \omega_{c} t, \sigma_{z}(t)=\sigma_{z} \cos \omega_{c} t+$ $\sigma_{x} \sin \omega_{c} t$, and $\sigma_{y}(t)=\sigma_{y}$ where the Larmor frequency 
$\omega_{c}=2 p_{x} \lambda / \hbar$. The spin $\sigma_{z}(t)$ varies with time and the spin current is always along the $x$ direction, $\left\langle\mathbf{j}_{s}^{z}\right\rangle=\frac{\hbar}{2} \frac{p_{x}}{m}\left(\left\langle\sigma_{z}\right\rangle \times\right.$ $\left.\cos \omega_{c} t+\left\langle\sigma_{x}\right\rangle \sin \omega_{c} t\right) \hat{x}$ where $\langle\ldots\rangle$ means the expectation value over an initial state $\langle r \mid \Phi\rangle$. As a result the spin transverse force on the spin is always perpendicular to the $x$ direction. Correspondingly the kinetic velocity $v_{x}$ and $v_{y}$ at a time $t$ are

$$
\begin{aligned}
& \left\langle v_{x}\right\rangle_{t}=\frac{p_{x}}{m}+\lambda\left\langle\sigma_{y}\right\rangle ; \\
& \left\langle v_{y}\right\rangle_{t}=-\lambda\left(\left\langle\sigma_{x}\right\rangle \cos \omega_{c} t-\left\langle\sigma_{z}\right\rangle \sin \omega_{c} t\right),
\end{aligned}
$$

respectively. Though $p_{y}=0$ the kinetic velocity $\left\langle v_{y}\right\rangle_{t}$ oscillates with the frequency $\omega_{c}$ while $\left\langle v_{x}\right\rangle_{t}$ remains constant. The $y$ component of the position is

$$
\langle y\rangle_{t}=\langle y\rangle_{t=0}-\frac{\hbar}{p_{x}} \sin \frac{\omega_{c} t}{2}\left(\left\langle\sigma_{x}\right\rangle \cos \frac{\omega_{c} t}{2}-\left\langle\sigma_{z}\right\rangle \sin \frac{\omega_{c} t}{2}\right) .
$$

If the initial state is polarized along the $y$ direction the electron spin does not vary with time as it is an energy eigenstate of the system, as discussed by Datta and Das [2]. In this case the spin current $\left\langle\mathbf{j}_{s}^{z}\right\rangle$ carried by the electron is always zero and the spin transverse force is zero. Thus $\left\langle v_{y}\right\rangle_{t}=0$. If the initial state is along the spin $z$ direction at $t=0$, i.e., $\left\langle\sigma_{z}\right\rangle=s= \pm 1$, it is found that $\left\langle v_{y}\right\rangle_{t, s}=$ $-s \lambda \sin \omega_{c} t$. Different spins will move in opposite directions. It can be understood that the spin precession makes the spin current whose polarization is projected along the electric field changes with time such that the spin force along the $y$ direction also oscillates with the frequency $\omega_{c}$. This force will generate a nonzero velocity of electron oscillating along the $y$ direction. Though $\left\langle v_{y}\right\rangle_{t, s=1}-$ $\left\langle v_{y}\right\rangle_{t, s=-1}=-2 \lambda \sin \omega_{c} t$, the velocity does not contribute to the spin current along the $y$ direction, i.e., $\left\langle\left\{v_{y}, \sigma_{z}\right\}\right\rangle_{t, s}=$ 0 . The trajectory oscillates with time. The amplitude of the oscillation is $\hbar / p_{x}$ and the frequency is $\omega_{c}=2 p_{x} \lambda / \hbar$. For a typical two-dimensional electron gas the electron density is $n_{e}=10^{11} \sim 10^{12} / \mathrm{cm}^{2}$ and the wavelength near the Fermi surface $\hbar / p_{x} \sim 3-10 \mathrm{~nm}$. For a typical Rashba coupling $\lambda=10^{-4} c, \omega_{c}=0.3 \sim 1.0 \times 10^{-14} \mathrm{~s}$. The rapid oscillation of the electron wave packet is known in the literature as the Zitterbewegung of an electron as a relativistic effect, which is usually regarded as a result of admixture of the positron state in an electron wave packet as a relativistic effect [12]. In semiconductors the Rashba coupling reflects the admixture of the particle and hole states in the conduction and valence bands. Recently Schliemann et al. obtained the solution of the trajectory and proposed that this effect can be observed in III-V zinc-blend semiconductor quantum wells [13]. In the $p$-doped semiconductors described by the Luttinger model there also exists a spin force, and will generate the Zitterbewegung as calculated by Jiang et al. [14]. Though the spin transverse force on a moving spin is very analogous to the Lorentz force on a moving charge, because of spin precession, its effect is completely different with the motion of a charged particle in a magnetic field, where the amplitude of the Lorentz force is constant and the charged particle moves in a circle. The Zitterbewegung of the electronic wave package near the boundary will cause some edge effect as shown in recent numerical calculations [15]. The edge effect is determined by the electron momentum. The smaller the momentum, the larger the edge effect. The amplitude and frequency of the Zitterbewegung satisfy a relation that $\left(\hbar / 2 p_{x}\right)\left(2 p_{x} \lambda / \hbar\right)=\lambda$, which is the amplitude of oscillation of the velocity $v_{y}$. In Fig. 1 it is illustrated that two electrons with different spins experience opposite forces in an electric field.

Furthermore we consider a two-dimensional electron gas lacking both the bulk and structural inversion symmetries. A Dresselhaus term $\beta\left(\mathbf{p}_{x} \sigma_{x}-\mathbf{p}_{y} \sigma_{y}\right)$ will be included in the total Hamiltonian in Eq. (8) [16]. In this model the spin force formula gives [17]

$$
\left\langle F_{f}\right\rangle=\frac{4 m^{2}}{\hbar^{2}}\left(\lambda^{2}-\beta^{2}\right)\left(j_{s}^{z} \times \hat{z}\right)
$$

for each moving electron. First of all, the force disappears at the symmetric point of $\lambda= \pm \beta$. At this point the operator $\sigma_{x} \pm \sigma_{y}$ is a good quantum number and there is no spin flip in the system. For $\lambda \neq \pm \beta$, the moving electron will experience a spin-dependent force and the force will change its sign near $\lambda=\beta$. A heuristic picture from this formula is that when a nonzero spin current $j_{s}^{z}$ goes through this system the spin-orbit coupling exerts the spin transverse force on the spin current, and drives electrons to form a charge Hall current perpendicular to the spin current. The injected spin current can be generated in various ways, such as by the spin force $\nabla(\mu \cdot \mathbf{B})[8]$ and circularly or linearly polarized light injection $[18,19]$. For instance we assume the spin current $j_{s}^{z}$ is generated by the linear polarized light injection which is proportional to the transition rate from the valence band to the conduction band with finite momentum and the life time of electrons at the excited states. In the relaxation time $\tau$ approximation in a steady state the drift velocity orthogonal to the spin current is $\left\langle v_{y}\right\rangle=\frac{4 m}{\hbar^{2}}\left(\lambda^{2}-\beta^{2}\right) j_{s}^{z} \tau$ if $\tau$ is not so long, i.e., $2 p_{x} \sqrt{\lambda^{2}+\beta^{2}} \tau \ll \hbar$. This nonzero

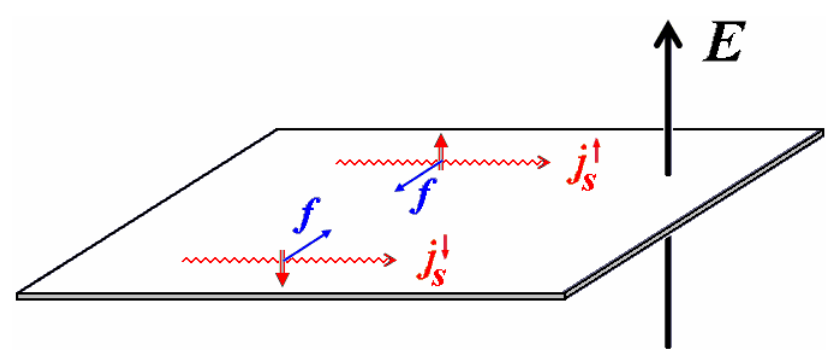

FIG. 1 (color online). The electric field exerts opposite forces on electrons with different spins polarized along the field. 
drift velocity will form a Hall current orthogonal to the spin current. This is the charge Hall effect driven by the spin current. In ferromagnetic metal or diluted magnetic semiconductors the charge current is spin polarized; it can generate the spin polarized Hall current via the spin transverse force. Thus the spin transverse force can also be regarded a driven force of an anomalous Hall effect [20] and the spin-resolved Hall effect [17,21]. A detailed calculation for this Hall conductance is given by the Kubo formula as a linear response to the field $B=\Delta B x \hat{z}$. This field will generate a spin force, $-\nabla\left(g \mu_{B} B \sigma\right)=$ $-g \mu_{B} \Delta B \sigma_{z} \hat{x}$, which will circulate a spin current along the $x$ direction, and, furthermore, the spin-orbit coupling provides a driving force to generate a transverse charge current, $j_{c, y}$. In the clear limit the Hall conductance $\sigma_{x y}=$ $j_{c, y} /\left(g \mu_{B} \Delta B\right)=0 \quad$ for $\quad \lambda= \pm \beta \quad$ and $(e / 4 \pi \hbar) \times$ $\left(\lambda^{2}-\beta^{2}\right) /\left|\lambda^{2}-\beta^{2}\right|$. However, following Inoue et al. and Mishchenko et al. [22] the inclusion of impurities scattering will suppress the Hall conductance completely just like the spin Hall effect. On the other hand numerical calculation in mesoscopic systems shows the existence of the effect [19]. Another example is the two-dimensional $p$-doped system with the cubic Rashba coupling [23],

$$
H=\frac{\mathbf{p}^{2}}{2 m}+i \alpha\left(\mathbf{p}_{+}^{3} \sigma_{-}-\mathbf{p}_{-}^{3} \sigma_{+}\right)
$$

where $\sigma_{ \pm}$are spin increasing and decreasing operators, and $\mathbf{p}_{ \pm}=\mathbf{p}_{x} \pm i \mathbf{p}_{y}$. The spin force on the moving electron in this system is $F_{f}=(2 m \mathbf{p} \alpha / \hbar)^{2}\left(\mathbf{j}_{s}^{z} \times \hat{z}\right)$. The linear response theory gives the Hall conductance $-9 e /(2 \pi \hbar)$ which is robust against the vertex correction from impurities scattering. Calculations by means of the GreenKeldysh function technique and linear response theory $[8,19]$ show the existence of charge Hall effect driven by the spin current, and the Onsager relation between the charge Hall effect and its reciprocal. The key features of the numerical results are in good agreement with the picture of spin force qualitatively.

In conclusion, an electric field exerts a transverse force on a moving spin just like a magnetic field exerts a Lorentz force on a moving charge. This force is proportional to the square of electric field and the spin current with spin projected along the field. This is a purely relativistic quantum mechanical effect. As the origin of the force the spin current should be also observable physically. From the solution of the motion of a single electron in an electric field, the Zitterbewegung of electronic wave packet in the spin-orbit coupling can be regarded as an explicit consequence of this force. Because of the similarity of this spin transverse force and the Lorentz force, the spin transverse force plays a similar role in the formation of the charge Hall effect driven by the spin current and the spin Hall effect driven by the charge current as the Lorentz force does in the Hall effect in a magnetic field.
The author thanks F. C. Zhang for helpful discussions. This work was supported by the Research Grant Council of Hong Kong (No. HKU 7039/05P), and a CRCG grant of The University of Hong Kong.

[1] G. A. Prinz, Science 282, 1660 (1998); S. A. Wolf et al., Science 294, 1488 (2001); Semiconductor Spintronics and Quantum Computation, edited by D. Awschalom, D. Loss, and N. Samarth (Springer, Berlin, 2002).

[2] Datta and Das, Appl. Phys. Lett. 56, 665 (1990).

[3] M. I. D'yakonov and V.I. Perel, JETP Lett. 13, 467 (1971); J.E. Hirsch, Phys. Rev. Lett. 83, 1834 (1999); J. Sinova et al., ibid. 92, 126603 (2004); S. Murakami, N. Nagaosa, and S. C. Zhang, Science 301, 1348 (2003); S. Q. Shen et al.,, Phys. Rev. Lett. 92, 256603 (2004).

[4] The Hall Effect and its Applications, edited by C. L. Chien and C. R. Westgate (Plenum, New York, 1980).

[5] J. Anandan, Phys. Lett. A 138, 347 (1989); R. C. Casella and S. A. Werner, Phys. Rev. Lett. 69, 1625 (1992); A. V. Balatsky and B.L. Altshuler, Phys. Rev. Lett. 70, 1678 (1993); C. M. Ryu, Phys. Rev. Lett. 76, 968 (1996).

[6] R. Winkler, Spin-Orbit Coupling Effects in TwoDimensional Electron and Hole Systems (Springer, Berlin, 2003).

[7] L. P. Rokhinson et al., Phys. Rev. Lett. 93, 146601 (2004).

[8] P. Zhang et al., cond-mat/0503505.

[9] E. I. Rashba, Fiz. Tverd. Tela (Leningrad) 2, 1224 (1960); [Sov. Phys. Solid State 2, 1109 (1960)].J. Luo et al., Phys. Rev. B 38, R10142 (1988).

[10] J. Nitta et al., Phys. Rev. Lett. 78, 1335 (1997).

[11] S. Q. Shen, Phys. Rev. B 70, 081311(R) (2004).

[12] E. Schrodinger, Sitzungsber. Preuss. Akad. Wiss. Phys. Math. Kl. 24, 418 (1930); H. Feshbach and F. Villars, Rev. Mod. Phys. 30, 24 (1958).

[13] J. Schliemann, D. Loss, and R. M. Westervelt, Phys. Rev. Lett. 94, 206801 (2005).

[14] Z. F. Jiang et al., Phys. Rev. B 72, 045201 (2005).

[15] A. Reynoso et al., Phys. Rev. B 70, 235344 (2004); B. K. Nikolic, L. P. Zarbo, and S. Welack, Phys. Rev. B 72, 075335 (2005).

[16] D. Dresselhaus, Phys. Rev. 100, 580 (1955).

[17] J. Li, L. Hu, and S. Q. Shen, Phys. Rev. B 71, 241305(R) (2005).

[18] E. Ya. Sherman, A. Najmaie, and J.E. Sipe, Appl. Phys. Lett. 86, 122103 (2005).

[19] E. M. Hankiewicz et al., Phys. Rev. B 72, 155305 (2005).

[20] R. Karplus and J.M. Luttinger, Phys. Rev. 95, 1154 (1954); J. Smit, Physica (Amsterdam) 21, 877 (1955); L. Berger, Phys. Rev. B 2, 4559 (1970).

[21] E. N. Bulgakov et al., Phys. Rev. Lett. 83, 376 (1999).

[22] J. Inoue, G.E.W. Bauer, and L. W. Molenkamp, Phys. Rev. B 70, 041303(R) (2004); E. G. Mishchenko, A. V. Shytov, and B. I. Halperin, Phys. Rev. Lett. 93, 226602 (2004).

[23] R. Winkler, Phys. Rev. B 62, 4245 (2000); R. Winkler et al., ibid. 65, 155303 (2002); J. Schliemann and D. Loss, ibid. 71, 085308 (2005). 